\title{
Joint TOA and DOA Estimation Compliant with IEEE 802.15.4a Standard
}

\author{
Eva Lagunas ${ }^{* 1}$, Montse Nájar ${ }^{* * 2}$, Monica Navarro *3 \\ \# Department of Signal Theory and Communications, Universitat Politècnica de Catalunya (UPC) \\ C/ Jordi Girona, 1-3, Barcelona, Spain. \\ 1 eva.lagunas@upc.edu \\ 2 montse.najar@upc.edu \\ ${ }^{*}$ Centre Tecnològic de Telecomunicacions de Catalunya (CTTC) \\ Parc Mediterrani de la Tecnologia, Av. Canal Olímpic s/n, Castelldefels (Barcelona), Spain. \\ 3 monica.navarro@cttc.es
}

\begin{abstract}
Ultra-WideBand (UWB) technology offers the potential of achieving high ranging accuracy through signal TimeOf-Arrival (TOA) measurements, even in harsh enviroments due to its ability to resolve multipath and penetrate obstacles. This paper evaluates a joint TOA and DOA estimator compliant with the IEEE 802.15.4a Standard. The estimator proposed here is an adaptation of [12] to comply with the Standard specifications. The estimation is developed in the frequency domain in order to exploit the high accurate time-based measures of the UWB signals.
\end{abstract}

\section{INTRODUCTION}

The nature of the short-duration pulses used in UltraWideBand (UWB) technology offers several advantages over narrowband communications systems. This paper is focused in one of the most attractive application of UWB which is short range positioning, especially indoor. Accurate determination of the location of wireless devices forms the basis of many new and interesting applications. UWB is one of the promising technologies to implement ranging systems considering the accuracy provided in time measurements due to its large bandwidth [1].

An UWB signal is defined as a signal that possesses an absolute bandwidth larger than $500 \mathrm{MHz}$ or a relative bandwidth larger than $20 \%$. It can coexist with incumbent systems in the same frequency range due to its large spreading factor and low power spectral density.

The need of information on the distance between network devices was indeed one of the main reasons for the definition of the IEEE 802.15.4a Standard (namely Standard in the sequel) [2]. Therefore, the Standard has the main interest in providing communications and high precision ranging capability with low power consumption and ultra low cost structures.

Positioning systems can be divided into three main categories [3]: Time-of-Arrival (TOA), Direction-of-Arrival (DOA) and Signal-Strength based systems. Signal-Strength method

\footnotetext{
${ }^{1}$ This work was partially supported by the Departament d'Universitats, Recerca $i$ Societat de la Informació de la Generalitat de Catalunya, by the Catalan Government under grant 2009 SGR 891, by the Spanish Government under project TEC2008-06327- C03 (MULTI-ADAPTIVE) and by the European Commission under project NEWCOM++ (ICT-FP7-216715).
}

does not exploit the advantages of large bandwidth and is for this reason that this option is ruled out. The Angle-ofArrival (AOA) based positioning technique involve the use of antenna arrays. Due to the large bandwidth of the UWB signal, the number of paths may be very large, especially in indoor enviroments. Therefore, accurate angle estimation becomes very challenging due to scattering from objects in the enviroments. Moreover, time-based approaches can provide very precise location estimates, and therefore they are better motivated for UWB. In order to cope with these problems and to achieve robust and reliable localization accuracy even in challenging areas, here it is proposed a frequency domain strategy for the joint TOA and DOA estimation, both based on time measurements.

Most localization techniques for UWB signals are based on time domain approaches. Optimal correlation based TOA estimator is proposed in [4] but it suffers from high sampling constraints. To cope with these sampling requirements a viable alternative is proposed in [5] where an energy-based TOA estimator is developed using sub-Nyquist sampling rates. TOA estimation has also been addressed based on frequency domain processing. In this contex, high resolution spectral techniques can be used. Methods based on the separation of the signal and the noise subspaces [6] are not proper for low values of SNR. In [10] methods based on Minimum Variance (MV) are proposed for wireless positioning systems. In fact, high resolution UWB TOA estimations can be obtained with the periodogram spectral estimator [11].

Estimation of the DOA in UWB systems has been addressed also considering subspace techniques [7] but they are of high complexity involving eigenvalue decompositions. In [8] is considered the estimation of AOA based on temporal delays but suffers from constraints associated with sampling requirements. In a previous work, the authors exploited the fact that the differences in arrival times of an incoming signal at different antenna elements contain the angle information for a known array geometry [9]. The idea of working in the frequency domain was first introduced by the authors in [10] and applied to UWB technology in [11]. Here, the 
joint TOA and DOA estimator introduced in [12] is evaluated and adapted to comply with the Standard. Working in the frequency domain any spectral estimation technique can be applied in order to obtain a power delay profile defined as the signal energy distribution with respect to the propagation delays from which the TOA estimation is obtained. The spectral estimation is based on the periodogram aiming at a low complexity implementation and assumes no knowledge of the received pulse waveform.

The outline of the paper is as follows: Section II introduces the UWB signal model for nodes equipped with an antenna array taking into account the Standard specifications. Section III describes the joint TOA and DOA estimation scheme. Finally, numerical results and conclusions are discussed in Section IV and V, respectively.

\section{UWB SIGNAL MODEL}

The IEEE 802.15.4a Standard specification can be found in [2]. It has two kinds of PHY; Impulse-Radio UWB (IR-UWB) and Chip Spread Spectrum (CSS). Among them, IR-UWB is accepted as the baseline for ranging and communication. The frame format structure is shown in Fig. 1.

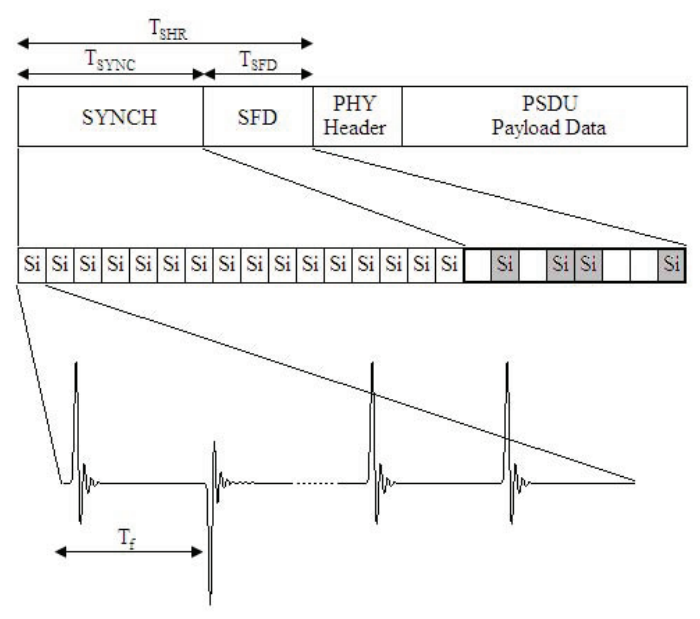

Fig. 1. Frame format structure

The Standard packet consists of a synchronization header (SHR) preamble, a physical layer header (PHR) and a data field (PSDU). The SHR preamble is composed of the ranging (SYNCH) preamble and the start of frame delimiter (SFD). The ranging preamble can consist of 16,64,1024,4096 symbols. The underlying symbol of the ranging preamble uses a length-31 ternary sequences, $c_{i}$. Each $c_{i}$ of length $N_{f}=31$ contains 15 zeros and 16 non-zero codes, and has the desired property of perfect periodic autocorrelation.

The mathematical model of the signal transmitted during the SHR is

$$
s(t)=\sum_{k=0}^{N_{S Y N C}+N_{S F D}-1} a_{k} \psi\left(t-k T_{s y m}\right)
$$

where $N_{S Y N C}$ and $N_{S F D}$ are the lengths of the SYNC and the SFD and $T_{\text {sym }}$ is the symbol duration. Coefficients $a_{k}$ are all equal to 1 during the SYNC while they take values $\{-1,0,+1\}$ during the SFD. Finally $\psi(t)$ is expressed as

$$
\psi(t)=\sum_{j=0}^{N_{f}-1} c_{i}(j) p\left(t-j T_{f}\right)
$$

In this equation $p(t)$ is an ultrashort pulse (monocycle), $T_{f}=$ $T_{\text {sym }} / N_{f}$ is the pulse repetition period and $c_{i}(j)$ denotes the $\mathrm{j}$-th element of sequence $c_{i}$.

Signal $s(t)$ propagates through an L-path fading channel whose response to $p(t)$ is $\sum_{l=0}^{L-1} h_{l} p\left(t-\tau_{l}\right)$. Note that it is assumed that the received pulse from each 1-th path exhibits the same waveform but experiences a different fading coefficient, $h_{l}$, and a different delay, $\tau_{l}$. Without loss of generality we assume $\tau_{0} \leq \tau_{1} \leq \ldots \leq \tau_{L-1}$, being $\tau_{0}$ the TOA that is to be estimated.

The received waveform at the q-th antenna element for a node equipped with an array of $Q$ antenna elements can be written as

$r_{q}(t)=\sum_{k=0}^{N_{S H R}-1} \sum_{j=0}^{N_{f}-1} \sum_{l=0}^{L-1} a_{k} c_{i}(j) h_{l, q} p\left(t-T_{k}^{j}-\tau_{l, q}\right)+w_{q}(t)$

where $w_{q}(t)$ is thermal noise with two-sided power spectral density $N_{o} / 2, T_{k}^{j}=j T_{f}+k T_{s y m}, h_{l, q}$ denotes the fading coefficient of the 1-th path at antenna q, and $N_{S H R}=$ $N_{S Y N C}+N_{S F D}$. Given the low duty cycle of UWB signals it is assumed non intersymbolic interference at the received signal. The temporal delay $\tau_{l, q}$ at each antenna element depends not only on the propagation delay but on the direction of arrival. Considering an uniform linear array (ULA), the propagation delay $\tau_{l, q}$ is given by

$$
\tau_{l, q}=\tau_{l}+q \frac{d}{c} \sin \left(\theta_{l}\right)
$$

with $d$ being the distance between antenna elements in the array, $c$ the speed of the light and $\theta_{l}$ the angle of arrival of the 1-th path. Although the scheme is presented assuming an ULA, it can be directly extended to other array configurations.

The signal associated to the $\mathrm{j}$-th transmitted pulse corresponding to the $\mathrm{k}$-th symbol, in the frequency domain yields

$$
Y_{j q}^{k}(w)=\sum_{l=0}^{L-1} a_{k} c_{i}(j) h_{l, q} S_{j}^{k}(w) e^{-j w \tau_{l, q}}+V_{j q}^{k}(w)
$$

with

$$
S_{j}^{k}(w)=\tilde{P}(w) e^{-j w\left(\left(k N_{f}+j\right) T_{f}\right.}
$$

where $\tilde{P}$ denotes the Fourier Transform of $\tilde{p}[n]$ which is the sampled received pulse waveform at the output of the Band Pass Filter (BPF) (see Fig. 2) and $N_{f}$ is the number of frames per symbol. $V_{j q}^{k}(w)$ is the noise in the frequency domain associated to the $\mathrm{j}$-th frame interval correspondig to the $\mathrm{k}$-th symbol. Sampling (5) at $w_{n}=w_{0} n$ for $n=0,1, \ldots, N-1$ 
where $w_{0}=\frac{2 \pi}{N T_{s}}$ and rearranging the frequency domain samples $Y_{j q}^{k}[n]$ into the vector $\mathbf{Y}_{j q}^{k} \in \mathbb{C}^{N \times 1}$ yields

$$
\mathbf{Y}_{j q}^{k}=\sum_{l=0}^{L-1} a_{k} c_{j} h_{l, q} \mathbf{S}_{j}^{k} \mathbf{e}_{\tau_{l, q}}+\mathbf{V}_{j q}^{k}=a_{k} c_{j} \mathbf{S}_{j}^{k} \mathbf{E}_{l, q} \mathbf{h}_{q}+\mathbf{V}_{j q}^{k}
$$

where the matrix $\mathbf{S}_{j}^{k} \in \mathbb{C}^{N \times N}$ is a diagonal matrix whose components are the frequency samples of $S_{j}^{k}(w)$ and the matrix $\mathbf{E}_{l, q} \in \mathbb{C}^{N \times L}$ contains the delay-signature vectors associated to each arriving delayed signal

$$
\mathbf{E}_{l, q}=\left[\begin{array}{lllll}
\mathbf{e}_{\tau_{0, q}} & \ldots & \mathbf{e}_{\tau_{j, q}} & \ldots & \mathbf{e}_{\tau_{L-1, q}}
\end{array}\right]
$$

with $\mathbf{e}_{\tau_{j, q}}=\left[\begin{array}{llll}1 & e^{-j w_{0} \tau_{j, q}} & \ldots & e^{-j w_{0}(N-1) \tau_{j, q}}\end{array}\right]^{T}$. The channel fading coefficients are arranged in the vector $\mathbf{h}_{q}=$ $\left[\begin{array}{lll}h_{0, q} & \ldots & h_{L-1, q}\end{array}\right]^{T} \in \mathbb{C}^{L \times 1}$, and the noise samples in vector $\mathbf{V}_{j q}^{k} \in \mathbb{C}^{N \times 1}$.

\section{JOINT TOA AND DOA ESTIMATION}

This section adapts the proposed frequency domain TOA and DOA estimator introduced by the authors in [12] to the Standard specifications. The modifications are mainly in the coarse stage due to the Time Hopping sequence removal by the new Standard. The block diagram of the frequency domain TOA and DOA estimator is sketched in Fig. 2.

\section{A. TOA Estimation}

The algorithm needs a measure of the TOA at each array element in order to obtain the final joint TOA and DOA estimation. This section describes how the estimation of the TOA is performed from the noisy observations $r_{q}(t)$ without the specific knowledge of the pulse shape $\tilde{p}(t)$. For the purpose of describing the estimation algorithm the receiver assumes an ideal Band Pass Filter (BPF) sampled at Nyquist rate, followed by a Discrete Fourier Transform (DFT) module that transforms the signal to the frequency domain. The frequency samples are processed without knowledge of the specific pulse shape in the fine TOA estimator stage. The fine estimator requires a previous stage that provides a coarse symbol synchronization. Note that the proposed receiver scheme is a non-coherent receiver and it is implemented in digital form using Nyquist frequency rate.

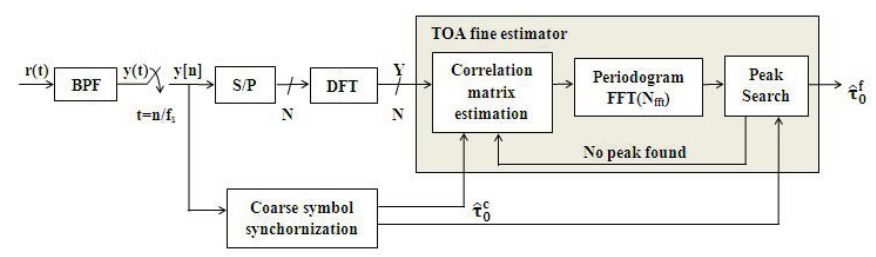

Fig. 2. Block diagram of the non-coherent receiver block for a single antenna

The TOA estimation for a single antenna compliant with the Standard was first presented by the authors in [13]. First, a simple coarse estimation stage that provides the time reference for symbol synchronization and estimates the threshold used in the TOA algorithm is needed. After the synchronization stage, the signal is passed through a fine TOA estimation stage which provide the final TOA measurement. The TOA defined as $\tau_{0}$ indicates the beginning of the first complete symbol in the observation interval, being $0 \leq \tau_{0} \leq T_{\text {sym }}$.

1) Coarse TOA Estimation: The coarse estimation consists of an energy estimator and a simple search algorithm that identifies the beginning of the symbol by applying a minimum distance criterion. Since the signal structure in the Standard does not include a time hopping sequence, the minimum distance criterion is applied in this case based on the ternary sequence knowledge, $c_{i}$, at symbol level.

It is assumed that the acquisition begins at any point of the SYNCH preamble $t_{0}$ and lasts $K_{s}+1$ symbols. Note that the acquisition window duration is defined one symbol longer than the number of symbols considered for the fine timing estimation. Hence the minimum acquisition window shall be equivalent to two symbols duration in order to perform the fine estimation over a single symbol $K_{s}=1$.

To find the beginning of the next symbol the frame number which the first detected pulse belongs to is needed. Lets denote $y[m]=y\left(m T_{s}\right)$ the discrete-time received signal, where $T_{s}$ is the sampling period. The time domain samples of the received signal in the $\mathrm{i}$-th time interval of duration $T_{f}$ are defined as

$$
y_{\text {frame }, i}[n]=y\left[(i-1) K_{f}+n\right] \text { for } n=1, \ldots, K_{f}
$$

where $i=1, \ldots, N_{f}\left(K_{s}+1\right)$. Rearranging the time domain samples $y_{\text {frame }, i}[n]$ in the vector $\mathbf{y}_{\text {frame }, i} \in \mathbb{C}^{K_{f} \times 1}$, being $K_{f}=\left\lfloor T_{f} / T_{s}\right\rfloor$ the number of samples in a frame interval, the energy at each frame interval in one symbol period is obtained averaging for each of the $N_{f}$ frames, over all $K_{s}+1$ symbols in the acquisition interval. That is

$$
E_{\text {frame }, j}=\sum_{k=0}^{K_{s}}\left\|\mathbf{y}_{\text {frame }, j+k N_{f}}\right\|^{2} \quad j=1, \ldots, N_{f}
$$

Although the energy estimation is done in the temporal domain, it can be also obtained in the frequency domain. Then the algorithm searches the 16 maxima corresponding to the 16 frames containing pulses and estimates the ternary sequence $\hat{c}_{i}$. From the original ternary sequence $c_{i}$ it is defined the vector d as a vector composed of the 16 positions of the sequence $c_{i}$ containing \pm 1 . Then it is defined circulant matrix $\Delta_{\rho_{c_{i}}}$ whose rows contain the relative delays in number of frame intervals between two consecutive pulses within a symbol period. Each row is a shifted version of the previous one. More specifically, defining $\rho_{c_{i}}(n)=d(n+1)-d(n)-1$ for $n=1, \ldots, 15$ and $\rho_{c_{i}}(16)=N_{f}-d(16)+d(1)$ as the number of frames between two consecutive transmitted pulses, the circulant matrix $\Delta_{\rho_{c_{i}}}$ is given by

$$
\Delta_{\rho_{c_{i}}}=\left[\begin{array}{ccccc}
\rho_{c_{i}}(1) & \rho_{c_{i}}(2) & \ldots & \rho_{c_{i}}(15) & \rho_{c_{i}}(16) \\
\rho_{c_{i}}(2) & \rho_{c_{i}}(3) & \ldots & \rho_{c_{i}}(16) & \rho_{c_{i}}(1) \\
\vdots & \vdots & \ddots & \vdots & \vdots \\
\rho_{c_{i}}(16) & \rho_{c_{i}}(1) & \ldots & \rho_{c_{i}}(14) & \rho_{c_{i}}(15)
\end{array}\right]
$$


Hence, with the estimated ternary sequence $\hat{c}_{i}$ it is conformed the vector $\hat{\mathbf{d}}$ which contains the estimated pulses positions or, in other words, which contains the 16 positions of the estimated sequence $\hat{c}_{i}$ containing \pm 1 . Therefore, the relative distance between the 16 estimated positions of the pulses form the vector

$$
\Delta \mathbf{d}=\left[\begin{array}{lllll}
\hat{\rho}_{c_{i}}(1) & \hat{\rho}_{c_{i}}(2) & \ldots & \hat{\rho}_{c_{i}}(15) & \hat{\rho}_{c_{i}}(16)
\end{array}\right]
$$

If we denote the first pulse within the symbol with the number 1 , the second with the number 2 and so on until the sixteenth pulse with the number 16 , then the estimated number of the first detected pulse $\eta \in\{1, \ldots, 16\}$ is carried out by finding the closest row of $\Delta_{\rho_{c_{i}}}$ which provides lower mean square error with respect to $\Delta \mathbf{d}$

$$
\eta=\arg \min _{j=1, \ldots, 16}\left\|\Delta \mathbf{d}-\left.\Delta_{\rho_{c_{i}}}\right|_{j}\right\|^{2}
$$

where $\left.\Delta_{\rho_{c_{i}}}\right|_{j}$ denotes the $\mathrm{j}$-th row of the matrix $\Delta_{\rho_{c_{i}}}$. From the estimated pulse number $\eta$ it is estimated the frame number $v \in\left\{1, \ldots, N_{f}\right\}$ which the first detected pulse belongs to. That is

$$
v=\mathbf{d}(\eta)-\hat{\mathbf{d}}(1)+1
$$

Then the TOA coarse estimation can be directly identified as

$$
\widehat{\tau}_{0}^{c}=\left(N_{f}-v+1\right) T_{f}
$$

The temporal resolution of this estimation is a frame period $T_{f}$.

2) Fine TOA Estimation: Once the beginning of the symbol is coarsely estimated, working in the frequency domain any type of spectral estimation can be applied to obtain a power profile defined as the signal energy distribution with respect to the propagation delays.

The fine TOA estimation $\hat{\tau}_{0}$ is obtained from the TOA coarse estimation $\hat{\tau}_{0}^{c}$ and a high resolution time delay $\tilde{\tau}$ estimate of the first arriving path with respect to the time reference obtained in the coarse estimation stage. The TOA estimation resulting from the fine estimation stage is given by

$$
\hat{\tau}_{0}=\hat{\tau}_{0}^{c}+\tilde{\tau}
$$

The TOA estimator consist of finding the first delay, $\tilde{\tau}$, that exceeds a given threshold, $P_{t h}$, in the power profile

$$
\tilde{\tau}=\min \arg _{\tau}\left\{P(\tau)>P_{t h}\right\}
$$

Given the signal frequency domain structure obtained in (7), the power delay profile can be obtained by estimating the energy of the frequency domain signal filtered by the delay signature vector, $\mathbf{e}_{\tau}=\left\{\begin{array}{llll}1 & e^{-j w_{0} \tau} & \ldots & e^{-j w_{0}(N-1) \tau}\end{array}\right\}^{T}$, at each time delay resulting in the quadratic form

$$
P(\tau)=\mathbf{e}_{\tau}^{H} \mathbf{R e}_{\tau}
$$

where $\mathbf{R}$ is the frequency domain signal correlation matrix computed from the received signal at each of the array antennas. Then $\mathbf{R} \in \mathbb{C}^{N \times N}$.
The quadratic form (18) allows for a low complexity implementation by applying the Fast Fourier Transform (FFT) to the following coefficients

$$
\tilde{R}_{n}=\left\{\begin{array}{l}
\sum_{k=n+1}^{N} \mathbf{R}_{k-n, k}: 0 \leq n \leq N-1 \\
\sum_{k=1}^{N+n} \mathbf{R}_{k-n, k}:-N+1 \leq n \leq 0
\end{array}\right\}
$$

where $\tilde{R}_{n}$ is the sum of the n-th diagonal elements of the correlation matrix $\mathbf{R}$. So, the maximization problem resorts to maximize the following expression

$$
P(\tau)=\sum_{n=1-N}^{-N+1} \tilde{R}_{n} e^{-j w_{0} \tau n}
$$

The number of points used in the DFT is equivalent to the number of values of $\tau$ to sweep. Therefore, the more points used more accurate will the TOA estimation be. This number of DFT points will be denoted as M.

In order to obtain a more robust estimation of the correlation matrix $\mathbf{R}$, it can be obtained averaging over $K_{s}$ symbols. The expression is

$$
\mathbf{R}=\frac{1}{N_{f} K_{s}} \sum_{k=1}^{K_{s}} \sum_{j=1}^{N_{f}} \mathbf{Y}_{j q}^{k} \mathbf{Y}_{j q}^{k}{ }^{H}
$$

Note that the computation of the correlation matrix also takes advantage of the inherent temporal diversity of the IRUWB signal, with $N_{f}$ repeated transmitted pulses for each information symbol, by computing the correlation matrix over the $N_{f}$ received frames.

\section{B. Joint TOA and DOA Estimation}

The proposed algorithm considers the use of TOA measurements at each antenna element in an antenna array for a joint estimation of TOA, $\tau_{0}$, and DOA, $\theta_{0}$. Once the previous TOA estimator is applied at each array element, the delay measurements are arranged in a vector $\mathbf{m}_{\tau}$

$$
\mathbf{m}_{\tau}=\left[\begin{array}{lll}
\hat{\tau}_{0,1} & \ldots & \hat{\tau}_{0, Q}
\end{array}\right]^{T}
$$

which depends on the TOA and on the DOA by the geometric relation (remember (4))

$$
\mathbf{m}_{\tau}=\mathbf{1} \tau_{0}+\mathbf{x} \sin \left(\theta_{0}\right)+\mathbf{n}
$$

where $\mathbf{1}=\left[\begin{array}{llll}1 & 1 & \ldots & 1\end{array}\right]^{T}, \mathbf{x}=\frac{d}{c}\left[\begin{array}{llll}0 & 1 & \ldots & Q-1\end{array}\right]^{T}$ contains the ordered indexes of the array antenna element, and $\mathbf{n}$ denotes the measurement noise vector. The measurement vector $\mathbf{m}_{\tau}$ can also be written as

$$
\mathbf{m}_{\tau}=\mathbf{Z b}+\mathbf{n}
$$

where $\mathbf{Z}=\left[\begin{array}{ll}\mathbf{1} & \mathbf{x}\end{array}\right]^{T}$ and $\mathbf{b}=\left[\begin{array}{ll}\tau_{0} & \psi\end{array}\right]^{T}$ is the vector of parameters to be estimated, with $\psi=\sin \left(\theta_{0}\right)$. Then, the best linear unbiased estimator [14] is given by

$$
\hat{\mathbf{b}}=\left(\mathbf{Z}^{T} \mathbf{Z}\right)^{-1} \mathbf{Z}^{T} \mathbf{m}_{\tau}
$$

Here, it is assumed that the noise associated to the measurements is white and Gaussian and has the same variance for all array elements (which is reasonable since the elements in the array experience similar propagation conditions). 


\section{NUMERICAL RESULTS}

For numerical evaluation of the algorithm we consider the channel models developed within the framework of the IEEE 802.15.4a. In particular it is used the CM1 Residential LOS channel model. The spatial dependency on the angle of arrival is introduced as in [9]- [12]. All simulations are given for 100 independent channel realizations. The main simulation parameters are shown in Table I. The pulse $p(t)=c(t) \cos \left(2 \pi f_{0} t\right)$ is a modulated 8th order Butterworth pulse with a $3 \mathrm{~dB}$ bandwidth of $1.3 \mathrm{GHz}$ and a center frequency $f_{0}=4.5 \mathrm{GHz}$ according to the European ECC. The pulse repetition period is $T_{f}=T_{\text {sym }} / N_{f}=128 \mathrm{~ns}$. The threshold level $P_{t h}$ used in the simulation is defined as the noise power level, which is estimated during the coarse estimation process.

TABLE I

SiMUlation PARAMETERS

\begin{tabular}{|c|c|}
\hline Parameter & Value \\
\hline Pulse duration $p(t), T_{p}$ & $0.77 \mathrm{~ns}$ \\
BandWidth $B W$ & $1.3 \mathrm{GHz}$ \\
Number of frames per symbol, PRF and $N_{f}$ & 31 \\
Number of symbols in the acquisition interval, $K_{s}$ & 47 symbols \\
Symbol duration, $T_{s y m}$ & $3974.4 \mathrm{~ns}$ \\
Sampling rate, $1 / T_{s}$ & $3 \mathrm{GHz}$ \\
Number of points of the DFT, N & 128 \\
Number of $\tau$ to be sweeped, M & 1024 \\
\hline
\end{tabular}

Considering an array equipped with two antennas in an scenario with a single source, Fig. 3 depicts the periodograms computed at each array element form which the TOA measurements are obtained.

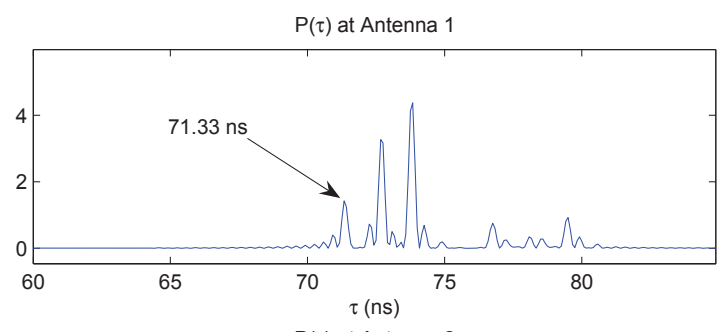

$\mathrm{P}(\tau)$ at Antenna 2

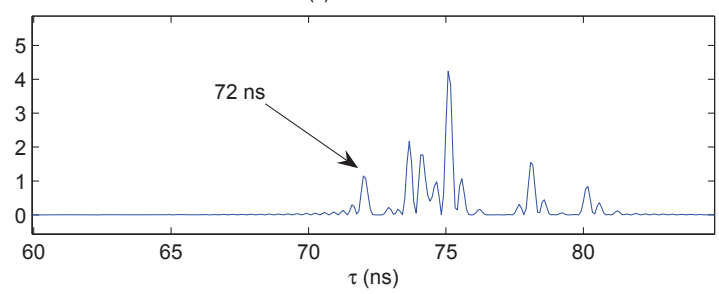

Fig. 3. Power delay profiles for an array equipped with two antennas with antenna elements separation of $36 \mathrm{~cm}$

The estimated TOA at each antenna element is given by the first peak that exceeds a given threshold corresponding to the first ray that gets to the array. These measurements yield the vector $\mathbf{m}_{\tau}$ used in the joint TOA and DOA estimator to obtain both estimated parameters.

One important step to get to the final result is the coarse symbol synchronization stage. In Fig. 4 it is showns the probability of failure of the synchronization stage with respect to the SNR for an array equipped with different number of antennas. One may observe that increasing the number of antennas $Q$ makes the coarse stage more robust due to the much more amount of information collected.

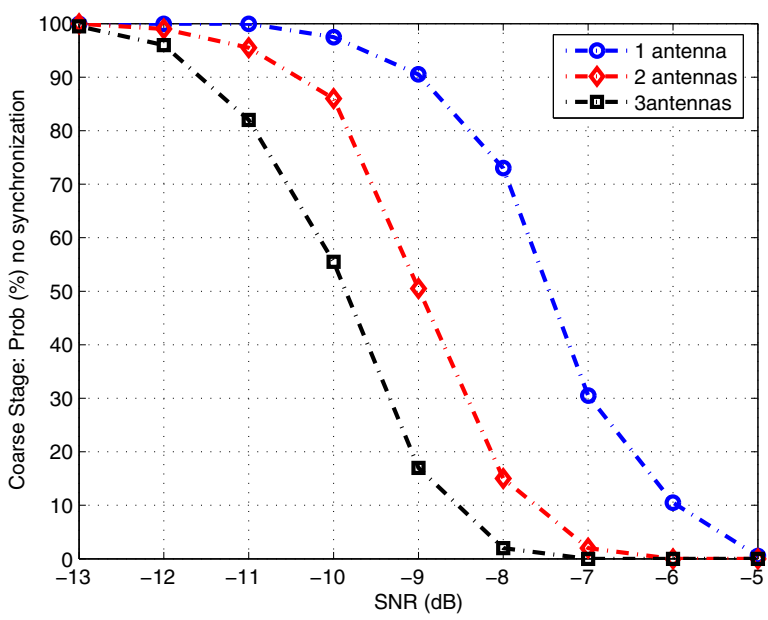

Fig. 4. Coarse Stage evaluation with respect to array size

Fig. 5 depicts the root mean squared error (RMSE) of the estimated TOA expressed in meters for arrays equipped with two and three antennas and an antenna spacing of $36 \mathrm{~cm}$. Results show that an estimation accuracy of few centimeters $(6 \mathrm{~cm})$ is asymptotically achieved for high values of SNR in both cases. The results are compared with the CRB derived for the joint TOA-DOA estimate [12]. The asymptotic behavior for high values of SNR is because the algorithm operates without knowledge of the pulse waveform and also because of the sampling frequency limitations.

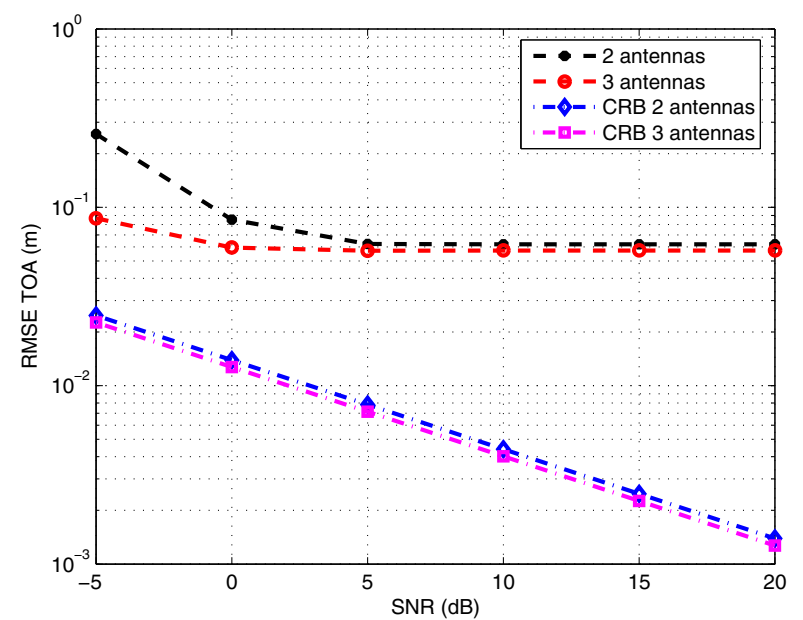

Fig. 5. Time of arrival $\tau_{0}$ estimation performance for 2 and 3 array elements with antenna elements separation of $36 \mathrm{~cm}$

Likewise, Fig. 6 shows the estimated sine of the DOA for the same scenario also compared with the CRB. We 
shall remark that the results depict directly the output of the estimator, that is the value of the sine of the DOA, rather than the angle itself. There is no need to transform to the angle domain for later application in positioning algorithms. One may observe that the error tends to increase while the SNR decreases.

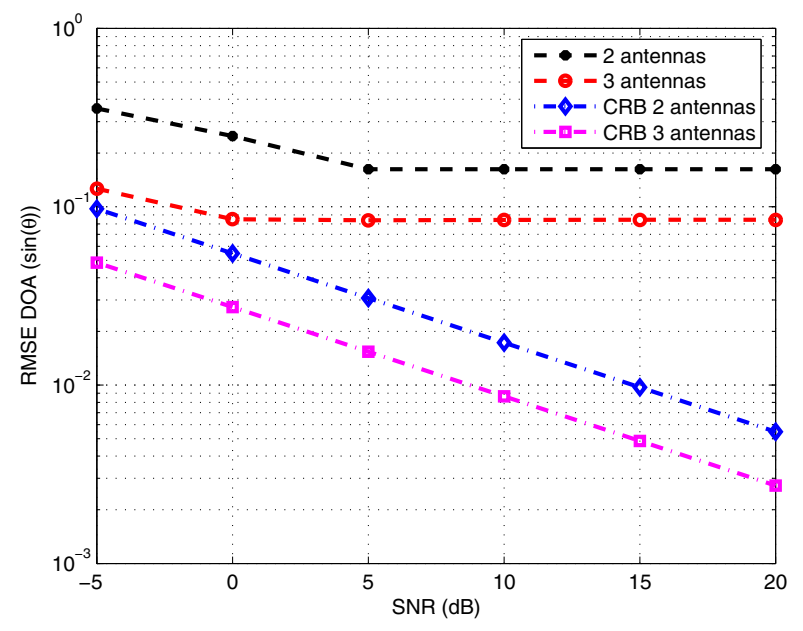

Fig. 6. Direction of arrival $\sin (\theta)$ estimation performance for 2 and 3 array elements with antenna elements separation of $36 \mathrm{~cm}$

This deterioration in the behavior of RMSE of both estimated parameters when SNR decreases happens when the noise level does not allow to properly detect the first arriving path and the algorithm takes as the first one that really is not (Fig. 7). In this case is obtained a wrong TOA estimation and the estimated DOA corresponds to the angle information of the multipath.
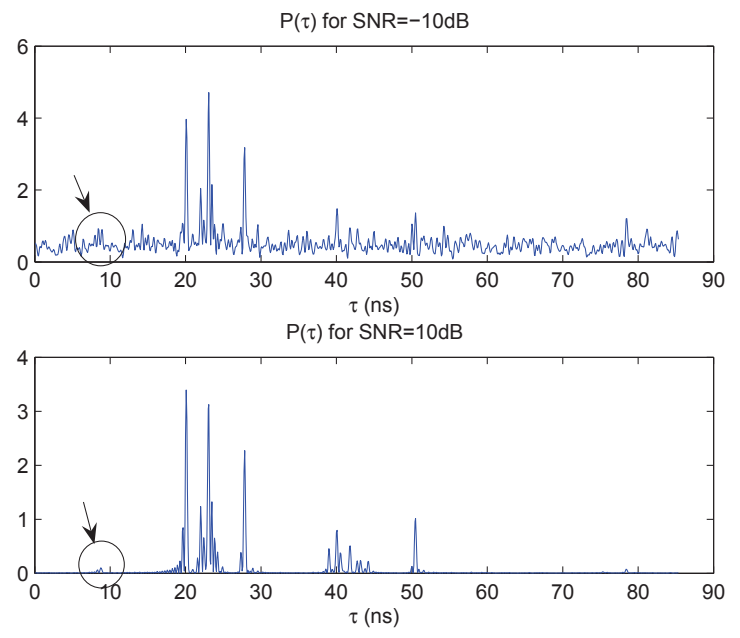

Fig. 7. Periodograms for the same antenna for $S N R=-10 d B$ and $S N R=10 d B$

\section{CONCLUSION}

A joint TOA and DOA estimator for UWB systems compliant with the IEEE 802.15.4a Standard has been presented.
The TOA is estimated at each array element in the frequency domain by means of a signal power delay spectrum based on the periodogram quadratic form. Its implementation becomes simple and efficient due to the DFT formulation. Finally both TOA and DOA estimations are obtained from the TOA measurements at each array antenna by means a linear estimation. The DOA estimation is able to exploit the high accuracy intrinsic to the large bandwidth of the UWB signal, since the angle estimation is performed from time delay measurements. Results have shown that only with two antenna element array accuracy of the order of $0.2 \mathrm{~ns}$ can be achieved for TOA estimation.

\section{REFERENCES}

[1] D. Porcino and W. Hirt, "Ultra-Wideband Radio Technology: Potential and Challenges Ahead", in IEEE Communications Magazine, pp. 66-74, July 2003.

[2] "802.15.4a-2007: IEEE Standard for Information Technology - Telecommunications and information exchange between systems - Local and metropolitan area networks - specific requirement. Part 15.4: Wireless Medium Access Control (MAC) and Physical Layer (PHY) Specifications for Low-Rate Wireless Personal Area Networks (WPANs)", publication date: 2007, page(s): 1-203, E-ISBN: 978-0-7381-5584-5, ISBN: 9780-7381-5538-8, INSPEC Accession Number: 9795136, Digital Object Identifier: 10.1109/IEEESTD.2007.4299496.

[3] D. Dardari, A. Conti, U. Ferner, A. Giorgetti and M. Win, "Ranging with Ultrawide Bandwidth Signals in Multipath Enviroments", in Proceedings of the IEEE, vol. 97, no. 2, pp. 404-537, Feb. 2009.

[4] E. Homier and R. Scholtz, "A Generalized Signal Flow Graph Approach for Hybrid Acquisition of Ultra-WideBand Signals", International Journal of Wireless Information Networks, vol. 10, no. 4, pp. 179-191, Oct. 2003.

[5] A.A. D'Amico, U. Mengali and L. Taponecco, "Energy-based TOA Estimation", IEEE Transactions on Wireless Communications, vol. 7, no. 3, pp. 1-10, March 2008.

[6] X. Li and K. Pahlavan, "Super-Resolution TOA Estimation with Diversity for Indoor Geolocation", IEEE Transactions on Wireless Communications, vol. 3, no. 1, pp. 224-234, Jan. 2004.

[7] H. Keshavarz,"Weighted Signal-Subspace Direction-Finding of UltraWideband Sources", IEEE International Conference on Wireless and Mobile Computing, Networking and Communications, WiMob'2005, Montreal, Canada, Aug. 22-24 2005, pp. 23-29.

[8] L. Pierucci and P.J. Roig, "UWB Localization on Indoor MIMO channels", IEEE International Conference on Wireless and Mobile Computing, Networking and Communications, WiMob'2005, Montreal, Canada, Aug. 22-24 2005, pp. 890-894.

[9] E. Lagunas, M. Nájar and M. Navarro, "UWB Joint TOA and DOA Estimation", Proceedings of IEEE International Conference on UltraWideband, ISBN 978-1-4244-2931-8 IEEE, pp.839-843, Vancouver, Canada, Sept. 9-11 2009

[10] J. Vidal, M. Najar and R. Jativa, "High Resolution Time-of-Arrival Detection for Wireless Positioning Systems", in the IEEE Vehicular Technology Conference, Vancouver, Canada, Sep. 2002.

[11] M. Navarro, S. Prior and M. Najar, "Low Complexity Frequency Domain TOA Estimation for IR-UWB Communications", in the IEEE Vehicular Technology Conference, Montreal, Canada, Sep. 2006.

[12] M. Navarro and M. Najar, "Frequency Domain Joint TOA and DOA estimation in IR-UWB", submitted to the IEEE Transactions on Wireless Communications, Oct. 29, 2008

[13] E. Lagunas, L. Taponecco, M. Nájar and A. D'Amico, "TOA Estimation in UWB: Comparison between Time and Frequency Domain Processing", submitted to the The 2nd International Conference on Mobile Lightweight Wireless Systems, Barcelona, Spain, May 10-12 2010.

[14] M. Kay, "Fundamentals on Statistical Signal Processing - Estimation Theory", Prentice Hall, 1993. 